\title{
Optical sum in Nearly Antiferromagnetic Fermi Liquid Model
}

\author{
E. Schachinger 1 , 因 and J.P. Carbotte ${ }^{2}$ \\ ${ }^{1}$ Institute of Theoretical and Computational Physics \\ Graz University of Technology, A-8010 Graz, Austria \\ ${ }^{2}$ Department of Physics and Astronomy, McMaster University, \\ Hamilton, Ontario, L8S 4 M1 Canada
}

(Dated: November 14, 2018)

\begin{abstract}
We calculate the optical sum (OS) and the kinetic energy (KE) for a tight binding band in the Nearly Antiferromagnetic Fermi Liquid (NAFFL) model which has had some success in describing the electronic structure of the high $T_{c}$ cuprates. The interactions among electrons due to the exchange of spin fluctuations profoundly change the probability of occupation $\left(n_{\mathbf{k}, \sigma}\right)$ of states of momentum $\mathbf{k}$ and spin $\sigma$ which is the central quantity in the calculations of OS and KE. Normal and superconducting states are considered as a function of temperature. Both integrals are found to depend importantly on interactions and an independent electron model is inadequate.

PACS numbers: $74.20 . \mathrm{Mn} 74.25 . \mathrm{Gz} 74.72 .-\mathrm{h}$
\end{abstract}

\section{INTRODUCTION}

The high $T_{c}$ oxides fall in the category of highly correlated systems. A manifestation of this fact is that in the underdoped regime there exists a

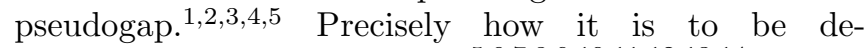
scribed remains controversial 5.6 .7 .8 .9 .10 .11 .12 .13 .14 In certain theories it is closely related to superconducting correlations, 5.6.7.8 and the superconducting transition temperature $T_{c}$ is the temperature at which phase coherence is lost. In other theories the pseudogap has its origin in completely different correlations and is a manifestation of a competing order such as a $d$-density wave (DDW) $9.10,11,12,13,14$ In any case interactions among charge carriers play an important role in such systems and cannot be ignored in any realistic approach to their properties even around optimum doping which is the case of interest here.

While many aspects of the superconducting state can be understood qualitatively on the basis of extensions of BCS theory, particularly around optimum doping, the search for essential differences 15 has remained an important avenue of investigation. In particular the idea of kinetic energy as opposed to potential energy driven superconductivity (i.e.: the kinetic energy is reduced at the transition temperature) and its relation to the OS has recently been given serious consideration in theory $15,16,17,18,19,20,21,22,23,24$ and experimentally 25.26 .27 .28 .29 .34 An issue of importance is the relation of the kinetic energy $(\mathrm{KE})$ to the optical sum (OS). A recent paper ${ }^{29}$ has provided some insight into this relationship and has given a comparison with experiment for the temperature variation of the OS in the normal state, its change at $T_{c}$, and its further evolution in the superconducting state. Correlations beyond BCS pairing were not considered, however. There have also been several recent studies of the temperature dependence of the OS, experimentally for the system $\mathrm{LA}_{2-x} \mathrm{Sr}_{x} \mathrm{CuO}_{4}$, Ref. 30, and, theoretically, using other models $31,32,33$
Within a BCS model, the condensation is potential energy driven and, in fact, the KE increases because the probability of occupation of the state $\mathbf{k}\left(n_{\mathbf{k}}\right)$ becomes smeared at the Fermi energy by the opening of the gap. This translates into a decrease in the OS as compared with its normal state value. But this is opposite to the behavior obtained experimentally in Ref. 27. However, as mentioned, the theoretical discussion of Ref. 29 is based on a non interacting model in the normal state and includes pairing correlations in the superconducting state only at the level of BCS. More sophisticated formulations of the theory of superconductivity could give different results. Also, interactions can significantly modify the results even in the normal state, as has been demonstrated recently by Knigavko et al. ${ }^{35}$ in a simplified model in which the charge carriers are coupled to a single Einstein mode. While only the normal state was considered, it was found that boson hardening (softening) results in an increase (decrease) in the OS and that interactions play an important role in determining temperature dependences.

In this paper we study a tight binding model with an emphasis on the effect interactions can have on the OS, particularly on its temperature dependence and its relationship to the $\mathrm{KE}$. Here, the interactions among the charge carriers are treated in the Nearly Antiferromagnetic Fermi Liquid (NAFFL) model 36 A review of its main properties and successes is given in Ref. 36 . The model is phenomenological and falls into the general class of boson exchange models where the interaction between electrons proceeds through the exchange of spin fluctuations. 37 The imaginary part of the spin susceptibility replaces the phonon propagator of the classic electron-phonon Eliashberg theory. The spin susceptibility could be calculated from microscopic theory. More usually, however, it is fit to experimental data, specifically to NMR in Ref. 37 . The basic idea is that the doped metallic cuprates are near an antiferromagnetic phase boundary and that coupling to spin fluctuations is therefore strong. While there is no consensus as to the validity of such a model when applied to 
the oxides the NAFFL model has been widely discussed and has had considerable successes particularly in correlating superconducting properties,, 38.39 .40 .41 .42 .43 and has been used to give a detailed description of the optical properties 40.44.45.46.47.48.49 in the high $T_{c}$ oxides at optimum doping. In any case, the NAFFL mode ${ }^{50}$ provides a convenient and specific framework within which we can study the effect of correlations on the OS and on related properties.

In Sec. III we summarize the basic equations that are needed to compute the optical sum integral as well as the KE. They are a set of three coupled generalized Eliashberg equations written for any momentum $\mathbf{k}$ in the two dimensional $\mathrm{CuO}_{2}$ Brillouin zone. They involve renormalized Matsubara frequencies, the renormalized quasiparticle energies as well as the superconducting energy gap. The interaction between the charge carriers is mediated by the exchange of spin fluctuations and involve the spin susceptibility. Fast Fourier transforms (FFT) provide solutions which give a $d$-wave gap as observed in experiments. In Sec. III we apply our solutions to evaluate the probability of occupation of the state of momentum $\mathbf{k}$ and spin $\sigma\left(n_{\mathbf{k}, \sigma}\right)$ from which the OS and the KE follow. Their temperature dependence is studied. Comparison with the non interacting case is made and it is concluded that interactions can profoundly modify results. Different behaviors can result depending on the choice of microscopic parameters. In Sec. [V] we consider explicitly the superconducting state. Here, again, generalized Eliashberg equations give variations with $T$ which are considerably different from earlier BCS results. When the electron-exchange boson interaction is taken as temperature independent and independent of state, the OS is lower in the superconducting than in the normal state. Nevertheless, it can keep increasing with decreasing temperature in contrast to BCS where it was found to decrease. This increase can be traced to the underlying temperature dependence of the OS which depends on details of the band structure and interactions involved, for example on the model spin susceptibility. Further, if we take account of the low energy gaping of the spin susceptibility which is brought about by the superconducting transition (a process which is not operative in the normal state) the KE can be further decreased and there is an additional increase in the OS which can effectively increase faster than it does in the normal state. In this case the KE in the superconducting state with low energy gaping of the spin susceptibility can be less than in the normal state without low energy gaping. An important conclusion of our work is that the observation of a faster increase in the OS with decreasing temperature in the superconducting state than in the normal state cannot unambiguously be taken to be an indication of kinetic energy driven superconductivity in contrast to a recent claim by van der Marel et al ${ }^{29}$ In Sec. $\nabla$ we provide a brief conclusion.

We use units in which $\hbar=c=1$ throughout this paper.

\section{FORMALISM}

In the NAFFL model the interaction between holes proceeds through the exchange of spin fluctuations and the spin susceptibility $\chi(\mathbf{q}, \omega)$ plays a central role. The three Eliashberg equations for the renormalized frequencies $\tilde{\omega}\left(\mathbf{k}, i \omega_{n}\right)$, the energy renormalization $\xi\left(\mathbf{k}, i \omega_{n}\right)$ and the pairing energy $\phi\left(\mathbf{k}, i \omega_{n}\right)$ as a function of momentum $\mathbf{k}$ in the two dimensional $\mathrm{CuO}_{2}$ Brillouin zone, and of fermionic Matsubara frequencies $i \omega_{n}=i \pi T(2 n+1), n=$ $0, \pm 1, \pm 2, \ldots$ and the temperature $T$ are $e^{41.42 .43}$

$$
\begin{aligned}
\tilde{\omega}\left(\mathbf{k}, i \omega_{n}\right)= & \omega_{n}+T \sum_{m} \sum_{\mathbf{k}^{\prime}} \lambda_{S F}\left(\mathbf{k}-\mathbf{k}^{\prime}, i \omega_{n}-i \omega_{m}\right) \\
& \times \frac{\tilde{\omega}\left(\mathbf{k}^{\prime}, i \omega_{m}\right)}{D\left(\mathbf{k}^{\prime}, i \omega_{m}\right)} \\
\xi\left(\mathbf{k}, i \omega_{n}\right)= & -T \sum_{m} \sum_{\mathbf{k}^{\prime}} \lambda_{S F}\left(\mathbf{k}-\mathbf{k}^{\prime}, i \omega_{n}-i \omega_{m}\right) \\
& \times \frac{\epsilon_{\mathbf{k}^{\prime}}+\xi\left(\mathbf{k}^{\prime}, i \omega_{m}\right)}{D\left(\mathbf{k}^{\prime}, i \omega_{m}\right)} \\
\phi\left(\mathbf{k}, i \omega_{n}\right)= & -T \sum_{m} \sum_{\mathbf{k}^{\prime}} \lambda_{S F}\left(\mathbf{k}-\mathbf{k}^{\prime}, i \omega_{n}-i \omega_{m}\right) \\
& \times \frac{\phi\left(\mathbf{k}^{\prime}, i \omega_{m}\right)}{D\left(\mathbf{k}^{\prime}, i \omega_{m}\right)} .
\end{aligned}
$$

In Eqs. (11) $D\left(\mathbf{k}, i \omega_{n}\right)$ is given by

$$
D\left(\mathbf{k}, i \omega_{n}\right)=\tilde{\omega}^{2}\left(\mathbf{k}, i \omega_{n}\right)+\left[\epsilon_{\mathbf{k}}+\xi\left(\mathbf{k}, i \omega_{n}\right)\right]^{2}+\phi^{2}\left(\mathbf{k}, i \omega_{n}\right),
$$

and $\epsilon_{\mathbf{k}}$ is the charge carrier dispersion relation. In a tight binding model without the inclusion of the coupling to the spin fluctuations it is given by:

$$
\begin{aligned}
\epsilon_{\mathbf{k}}= & -2\left\{t\left[\cos \left(a k_{x}\right)+\cos \left(a k_{y}\right)\right]\right. \\
& \left.-2 t^{\prime} \cos \left(a k_{x}\right) \cos \left(a k_{y}\right)\right\}-\mu^{*} .
\end{aligned}
$$

Here, $a$ is the lattice parameter in the copper oxide plane, $t$ the nearest neighbor hopping, $t^{\prime}$ the next nearest neighbor hopping, and $\mu^{*}$ the chemical potential. We will discuss within this context, two tight binding models with the parameters given in Table! The corresponding Fermi surface is presented in Fig. 10 for model A and in Fig. 10 for model B. These figures define also the various points in the Brillouin zone. The dotted lines indicate the antiferromagnetic Brillouin zone boundary. For model A, the Fermi surface crosses the anti-ferromagnetic Brillouin zone around $X$ and symmetry related points. These points are 'hot spots' for which Fermi surface to Fermi surface transitions are possible with momentum transfer $(\pi / a, \pi / a)$ (nesting property). Model A has been used previously in Ref. 29 to discuss the OS and KE in $\mathrm{Bi}_{2} \mathrm{Sr}_{2} \mathrm{Cu}_{2} \mathrm{O}_{8+\delta}$ (BSCCO) assuming no interactions between the electrons in the normal state. Therefore, it is natural to employ this same model in the present study which extends the previous calculations to include exchange of spin fluctuations between charge carriers in the 
TABLE I: The two tight binding models used within this paper. Model A corresponds to the tight binding model discussed by van der Marel et al. ${ }^{29} t$ and $t^{\prime}$ are given in meV, the critical temperature $T_{c}$ in $\mathrm{K}$, and the filling $\langle n\rangle$ is defined in Eq. (9).

\begin{tabular}{lcccr}
\hline \hline Model & $t$ & $t^{\prime}$ & $\langle n\rangle$ & $T_{c}$ \\
\hline $\mathrm{A}$ & 148.8 & 40.9 & 0.425 & 90 \\
$\mathrm{~B}$ & 100.0 & 16.0 & 0.4 & 100 \\
\hline \hline
\end{tabular}

NAFFL model. The second model, Model B, was chosen to contrast with the first. It has no hot spots and its Fermi surface is closed around the $\Gamma$-point in contrast to Model A which has a Fermi surface which is closed around the $M$-point. It also has a smaller value of $t$ which, on its own, would imply a smaller absolute value of KE. These differences in band parameters lead, as we shall see, to some differences in KE and optical sum at $T=0$ in the normal state.

In the phenomenological model of Millis et al ${ }^{36.37}$ and

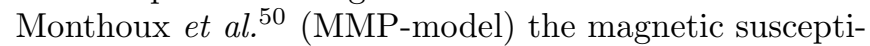
bility was fit to NMR data and its imaginary part takes on the form

$$
\Im m \chi_{M M P}(\mathbf{q}, \omega)=\frac{\chi_{\mathbf{Q}}\left(\omega / \omega_{S F}\right)}{\left[1+\zeta^{2}(\mathbf{q}-\mathbf{Q})^{2}\right]^{2}+\left(\omega / \omega_{S F}\right)^{2}},
$$

where $\chi_{\mathbf{Q}}$ is the static susceptibility, $\mathbf{Q}$ is the commensurate antiferromagnetic wave vector $(\pi / a, \pi / a)$ in the upper right hand quadrant of the $\mathrm{CuO}_{2}$-plane Brillouin zone and symmetry related points. $\zeta$ is the magnetic coherence length, and $\omega_{S F}$ a characteristic spin fluctuation frequency. We set $\zeta=2.5 a$ throughout this paper and various values for $\omega_{S F}$ are investigated.

The kernel $\lambda_{S F}\left(\mathbf{q}, i \nu_{n-m}\right)$ in Eqs. (1) with momentum transfer $\mathbf{q}=\mathbf{k}-\mathbf{k}^{\prime}$ and the bosonic Matsubara frequency $i \nu_{n-m}=i \omega_{n}-i \omega_{m}$ is given as

$$
\lambda_{S F}\left(\mathbf{q}, i \nu_{n}\right)=\frac{g^{2} \chi_{\mathbf{Q}}}{1+\zeta^{2}(\mathbf{q}-\mathbf{Q})^{2}+\left(\left|\nu_{n}\right| / \omega_{S F}\right)},
$$

with $g^{2} \chi_{\mathbf{Q}}$ adjusted to get the desired value of the critical temperature $T_{c}$ for a certain value of $\omega_{S F}$ from the solution of the linearized Eqs. (11). This defines the model.

The aim of this paper is to investigate the effect interactions have on the OS defined as

$$
\pi e^{2} I_{\sigma}=\int_{-\Omega}^{\Omega} d \omega \Re \mathrm{e} \sigma_{x x}(\omega)=\frac{\pi e^{2}}{V} \sum_{\mathbf{k}, \sigma} n_{\mathbf{k}, \sigma} \frac{\partial^{2} \epsilon_{\mathbf{k}}}{\partial k_{x}^{2}},
$$

where $e$ is the charge on the electron, $V$ the volume, and $n_{\mathbf{k}, \sigma}$ is the probability of occupation of a state of momentum $\mathbf{k}$ and spin $\sigma$. Finally, $\sigma_{x x}(\omega)$ is the optical conductivity. The integral in Eq. (6) is to be taken over the single band with $\Omega$, the upper limit in the integral of Eq. (6), large enough to include all possible transitions in that band. We are also interested in the relationship
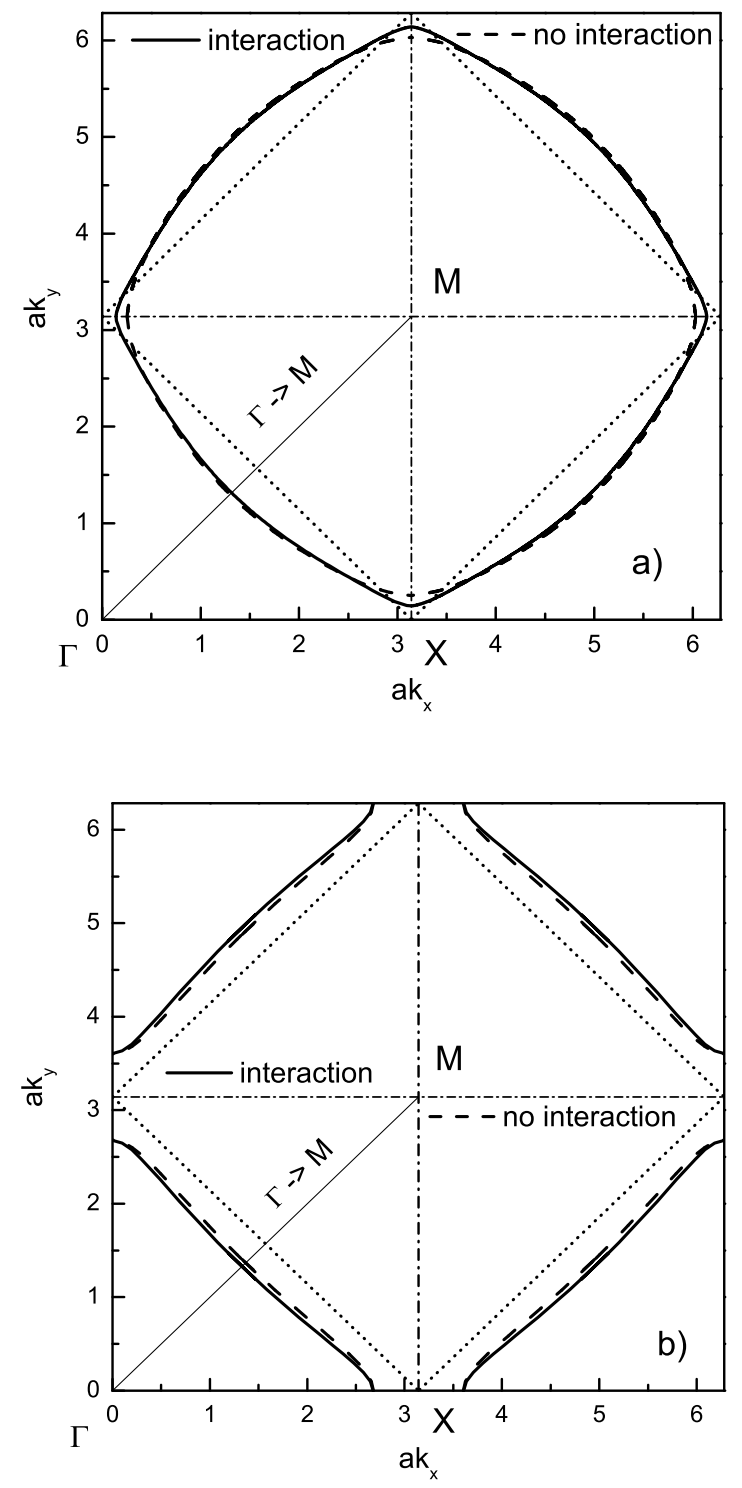

FIG. 1: The Fermi surface for the non interacting system (dashed line) and the system with interaction (solid line). a) model A of Table \ b) for model B.

between the OS and the kinetic energy. By definition

$$
I_{\mathrm{KE}}=\left\langle H_{\mathrm{KE}}\right\rangle=\frac{a^{2}}{V} \sum_{\mathbf{k}, \sigma} n_{\mathbf{k}, \sigma} \epsilon_{\mathbf{k}} .
$$

We will see that, to a good approximation $I_{\sigma}$ and $-I_{\mathrm{KE}}$ are nearly proportional to each other. Thus,

$$
\rho_{L} \propto \frac{1}{\pi e^{2}} \int_{-\Omega}^{\Omega} d \omega \Re \mathrm{e} \sigma_{x x}(\omega) \approx-\frac{1}{2}\left\langle H_{\mathrm{KE}}\right\rangle .
$$

holds approximately. (An equal sign would be appropriate if the dispersion relation (3) contained only nearest neighbor interaction, i.e.: $t^{\prime}=0$.) Here, $\rho_{L}$ is the experimentally determined value of the OS $\left(I_{\sigma}\right)$. 


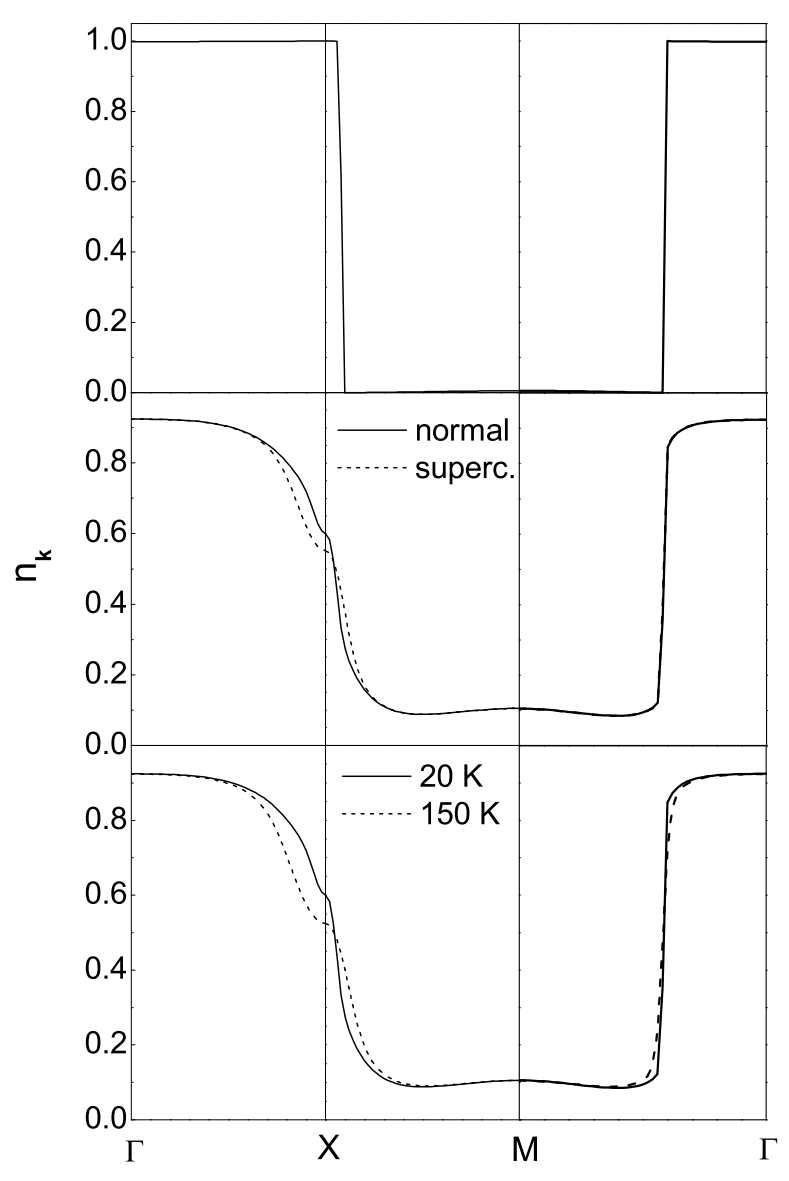

FIG. 2: The occupation number $n_{\mathbf{k}}$ for both spin states for selected directions in the $\mathrm{CuO}_{2}$ Brillouin zone. Model A of Table \was used. Top frame: the non interacting case. Center frame: The interacting case at a temperature $T=20 \mathrm{~K}$. We show normal state (solid line) and superconducting state (dashed line) results. Bottom frame: The temperature influence on the normal state $n_{\mathbf{k}}$ for $T=20 \mathrm{~K}$ (solid line) and $T=150 \mathrm{~K}$ (dashed line).

Interactions have a profound effect on the probability of occupation of the state $|\mathbf{k}, \sigma\rangle$ which would be one or zero for occupied and unoccupied states respectively in the non interacting case. In Fig. 22 we show results for $n_{\mathbf{k}}$, along certain selected directions in the $\mathrm{CuO}_{2}$ Brillouin zone. In all cases Model A of Table \is used for the electronic dispersion (3) with $\mu^{*}$ adjusted to the required filling which is is defined as

$$
\langle n\rangle=\frac{1}{2}-\sum_{\mathbf{k}} \sum_{n \geq 0} \frac{\epsilon_{\mathbf{k}}+\xi\left(\mathbf{k}, i \omega_{n}\right)}{\tilde{\omega}^{2}\left(\mathbf{k}, i \omega_{n}\right)+\left[\epsilon_{\mathbf{k}}+\xi\left(\mathbf{k}, i \omega_{n}\right)\right]^{2}+\phi^{2}\left(\mathbf{k}, i \omega_{n}\right)}
$$

and the charge carrier spin fluctuation strength $g^{2} \chi_{\mathbf{Q}}$ is adjusted to get a $T_{c}=90 \mathrm{~K}$ for the superconducting state. In the top frame of Fig. 2 we show $n_{\mathbf{k}}$ in the non interacting case as we go from $\Gamma$ to $X$ and from $X$ to $M$ in the Brillouin zone with the Fermi surface defined as the value of $\mathbf{k}$ at which $n_{\mathbf{k}}$ jumps from one to zero. It is obvious from Fig. Ta (dashed line) that the path $\overline{X M}$ crosses the Fermi surface. A second crossing of the Fermi surface can be observed along the path from $M$ to $\Gamma$.

The center frame of Fig. 22 shows $n_{\mathbf{k}}$ when interactions are taken into account. (The corresponding Fermi surface is shown as the solid line in Fig. [1.) We see a drastic difference in the value of $n_{\mathbf{k}}$ which is now of the order 0.9 at the $\Gamma$ point indicating that the effect of the interaction is very significant even in the center of the Brillouin zone. Also, $n_{\mathbf{k}}$ is of the order 0.1 outside the non interacting Fermi surface where $n_{\mathbf{k}} \approx 0$ for the non interacting case. The solid line applies to the normal state at $T=20 \mathrm{~K}$ and the dashed line to the superconducting state at the same temperature. On comparing these two cases we see that the transition to the superconducting state depletes even further the non interacting sea in the sense that it further reduces $n_{\mathbf{k}}$ at $\mathbf{k s}$ below the Fermi surface and correspondingly increases the probability of occupation of states right above the Fermi surface beyond the effect of interactions in the normal state. It is to be noted, however, that the onset of superconductivity results in rather modest changes in $n_{\mathbf{k}}$ as compared to the difference between interactions and no interactions in the normal state.

The bottom frame of Fig. 2 gives results in the normal state but compares two temperatures, namely $T=20 \mathrm{~K}$ (solid line) and $T=150 \mathrm{~K}$ (dashed line). Comparison with the middle frame shows that increasing the temperature has roughly the same qualitative effect on $n_{\mathbf{k}}$ as does the transition to the superconducting state. In both cases, the KE given by Eq. (77) increases because the states of lower $\epsilon_{\mathbf{k}}$ get depleted while states with higher $\epsilon_{\mathbf{k}}$ are occupied with increasing probability. This will also hold for the OS according to Eq. (6) which will now depend on interactions and on temperature.

\section{RESULTS FOR THE OPTICAL SUM IN THE NORMAL STATE}

In Fig. 3] we show results for the optical sum $\left(I_{\sigma}\right)$ Eq. (6) and compare with the kinetic energy $\left(I_{\mathrm{KE}}\right)$, Eq. (7). The top frame is based on Model A and the bottom frame on Model B of Table $\square$ The solid squares and circles are $-I_{\mathrm{KE}} / 2$ and $I_{\sigma}$ respectively in the free tight binding case, i.e.: no interactions, plotted as a function of the square of the temperature for the normal state. (A $128 \times 128$ sampling of the $\mathbf{k}$-space, $a k_{x}, a k_{y} \in[0, \pi]$, was used but going to a $256 \times 256$ sampling did not influ, ence the results.) In both models variation with $T$ over the range 0 to $200 \mathrm{~K}$ is small (of order $1 \%$ for Model A and $2 \%$ for Model B) as was also found in the work of Molegraaf et al $\Omega^{27}$ Also, the two integrals $\left(I_{\sigma},-I_{\mathrm{KE}} / 2\right)$ track each other closely even though they are not equal in magnitude. (They would be equal for $t^{\prime}=0$. We tried other Fermi surfaces, even one with perfect nesting, i.e.: $t^{\prime}=0$ and $\langle n\rangle=0.5$, and found no qualitative changes.) 


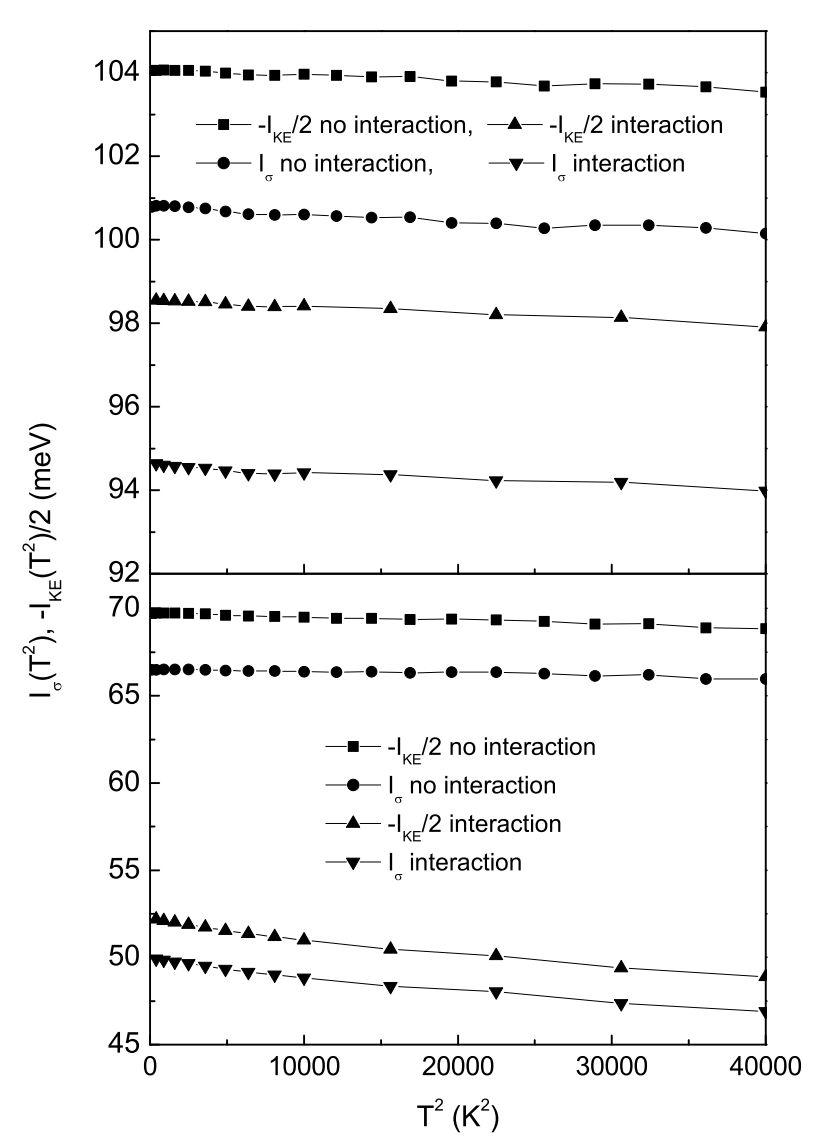

FIG. 3: Optical sum $I_{\sigma}$ and kinetic energy, $-I_{\mathrm{KE}} / 2$, as a function of $T^{2}$. Solid circles and squares are for the non interacting case while solid up-triangles and solid down-triangles include interactions. The top frame applies to Model A of Table \and $\omega_{S F}=82 \mathrm{meV}$ was used. Here, the interacting and non interacting cases show similar temperature dependencies. The bottom frame is for Model B of Table I with an MMP-model $\omega_{S F}=10 \mathrm{meV}$. Note the difference in temperature dependence between interacting and non interacting case.

These results are for comparison with results indicated by up/down-triangles which include interactions in the NAFFL model. The magnitude of both, the optical sum integral and the kinetic energy has been changed considerably by the interactions although the order remains the same, i.e.: $-I_{\mathrm{KE}} / 2$ is greater than $I_{\sigma}$ and, again, they track each other. More importantly, for the discussion here, the temperature variation has been changed. Both integrals now show variation of the order 8 to $9 \%$. Also the dependence on $T^{2}$ is not linear at small values of $T^{2}$. Model A shows similar behavior for small values of $\omega_{S F}$. It is clear that any estimate based on the independent particle tight binding model is unreliable. It is, however, possible to chose specific parameters in the MMP-model which show variations in the interacting case that are much closer to the non interacting case. This is illus- trated in the top frame of Fig. 3 Here we used Model A of Table 【 Again, results with and without interaction are compared and both show little temperature variation. To get this we used $\omega_{S F}=82 \mathrm{meV}$ in our MMP form of Eq. (4) without a change in the magnetic coherence length. Both results, with and without interaction, show little variation with temperature. What this shows is that the magnitude as well as the temperature variation of KE and of OS depends significantly on the parameters used to characterize their electronic structure, particularly the spin susceptibility.

We have done additional calculations for Model A with $\omega_{S F}=40,20$, and $10 \mathrm{meV}$. In all cases the change in $\mathrm{KE}$ due to interactions at $T=0$ increases with decreasing values of $\omega_{S F}$. In particular, it changes at $T=0$ by $5.3 \%$ when compared with the non interacting case, for $\omega_{S F}=82 \mathrm{meV}$ and by $15.8 \%$ for $\omega_{S F}=10 \mathrm{meV}$. The corresponding temperature changes from $T=0$ to $T=$ $200 \mathrm{~K}$ are roughly a factor of 5 smaller, more precisely, they are $0.7 \%$ and $3.4 \%$ respectively. Thus, a change in $\mathrm{KE}$ at $T=0$ due to interactions also implies a corresponding change in temperature dependence with both changes tracking each other. For Model B the change in $\mathrm{KE}$ due to interactions is $34 \%$ for $\omega_{S F}=10 \mathrm{meV}$ with a $8.7 \%$ increase in KE from $T=0$ to $T=200 \mathrm{~K}$. These variations are about a factor of two larger than for the equivalent case of Model A with a comparable value of $\omega_{S F}$. Despite the fact that the two models represent very different band structures $I_{\sigma}$ and $-I_{K E} / 2$ show essentially the same qualitative features in their temperature and $\omega_{S F}$ dependence. However, the quantitative differences are important.

\section{RESULTS FOR THE OPTICAL SUM IN THE SUPERCONDUCTING STATE}

Results for the superconducting state are illustrated in Fig. 4 which has two frames. The top frame applies to the band structure Model A of Table $\square$ and is for $\omega_{S F}=82 \mathrm{meV}$ as in the top frame of Fig. 3 We have also included $2 \%$ impurities in the unitary limit but this serves mainly to illustrate that impurities introduce no qualitative differences into our results. We see that, as we expect, superconductivity reduces the optical integral (open triangles) as compared with its normal state (solid triangles) value at the same temperature. This reduction is small. For the top frame which shows the least temperature dependence, the KE integral shows a reduction of about $0.25 \%$ below its normal state value which can be compared with the results shown in the bottom frame of Fig. 6 of Ref. 34 where the difference is $0.2 \%$ in their BCS calculations. On the other hand, in the bottom frame of

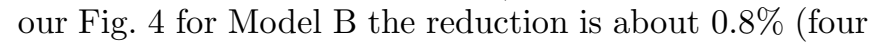
times larger). This shows that the Eliashberg results depend on band structure as well as on the details of the interactions involved, in particular on the value of $\omega_{S F}$. In the BCS limit the increase in KE normalized to the 


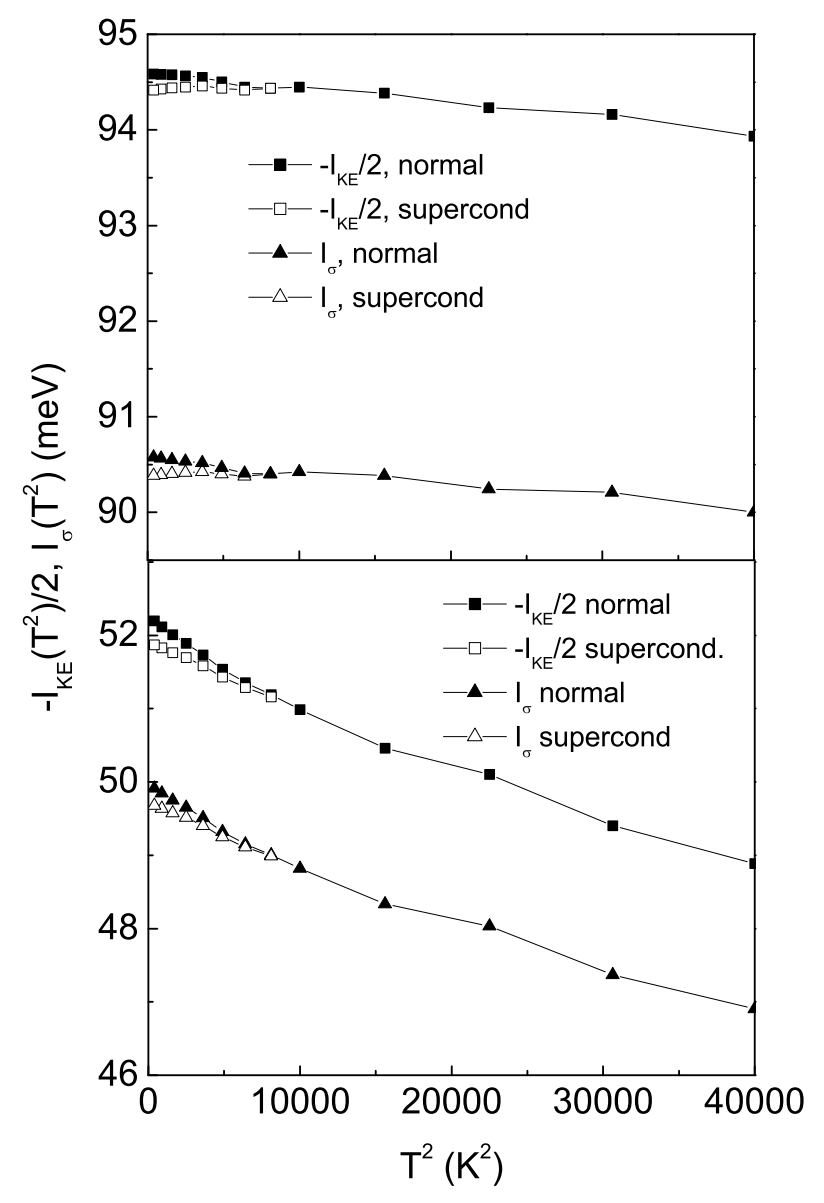

FIG. 4: Comparison of normal and superconducting state for the optical sum and the kinetic energy. The top frame applies to Model A of Table \and $\omega_{S F}=82 \mathrm{meV}$ and the bottom frame is for the band structure Model B of Table I and $\omega_{S F}=10 \mathrm{meV}$.

absolute value of the condensation energy is given by the formula $\left[\ln \left(\frac{\omega_{D}}{T_{c}}\right)-0.38\right]$ for both $s$ - and $d$-wave superconductors. Here $\omega_{D}$ is the Debye energy. This shows a strong dependence on $\omega_{D} / T_{c}$. The formula itself, however, is valid only for $\omega_{D} / T_{c} \gg 1$ and cannot be used to understand our Eliashberg results. The NAFFL model includes interactions which, as we have seen, change importantly the probability of occupation $n_{\mathbf{k}}$ and consequently the optical integral as well as the kinetic energy. For the parameters of Model B and $\omega_{S F}=10 \mathrm{meV}$ we find that $I_{\sigma}$ and $-I_{\mathrm{KE}} / 2$ can keep increasing with decreasing temperature in the superconducting state (bottom frame of Fig. (4). The open squares and triangles (superconducting state) are below their solid counterparts (normal state) but still keep growing as the temperatures is reduced. This does not indicate an exotic mechanism but comes directly from a generalization of Eliashberg theory that includes anisotropy in the band

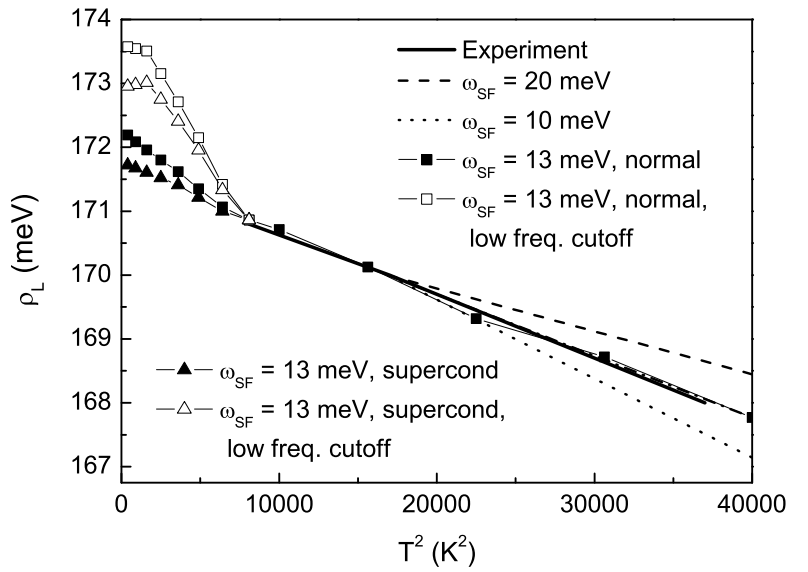

FIG. 5: The optical sum as a function of the square of the temperature for the band structure Model A of Table \with different values of $\omega_{S F}$. Also in one case a low frequency cutoff is applied to the spin susceptibility. Note the significance of the $T^{2}$-variation on the value of $\omega_{S F}$. The solid line indicates experimental normal state results reported by Molegraaf et $a l^{27}$

structure and, more importantly, the interaction due to coupling to the spin fluctuations.

In Fig. 5 we show additional results where the OS is seen to increase even more rapidly, with reduced temperature below the onset of superconductivity than it does in the normal state above $T_{c}$. What is shown is $\rho_{L}$ which is $I_{\sigma}$ or $-I_{\mathrm{KE}} / 2$ scaled to agree with experiment as discussed below; either, $I_{\sigma}$ or $-I_{\mathrm{KE}} / 2$, will do since these differ mainly by a different scaling factor. Only the OS is considered but the KE integral follows the same trend and therefore the optical measurement can again be used to get information on $\mathrm{KE}$ and its variation with temperature.

While in obtaining Fig. [5 we applied Model A which was used by van der Marel et al $\stackrel{29}{29}$ to describe their optimally doped and underdoped BSCCO samples, we now vary, in contrast to the top frame of Fig. [ the value of $\omega_{S F}$ used in the MMP-model for the spin susceptibility Eq. (4). Results are presented for $\omega_{S F}=20 \mathrm{meV}$ (dashed line), $10 \mathrm{meV}$ (dotted line), $13 \mathrm{meV}$ (solid squares) for the normal state, and solid triangles for the superconducting state. Also shown as the thick solid line are the experimental results of Ref. 27 for their optimally doped sample. We have scaled our theoretical results to agree with experiment at $T=120 \mathrm{~K}$. We first note that varying $\omega_{S F}$ in the normal state can strongly influence the temperature dependence obtained for $\rho_{L}$ (in $\mathrm{meV}$ ). The value of $\omega_{S F}=13 \mathrm{meV}$ was chosen from a best fit in the region $120 \mathrm{~K} \leq T \leq 200 \mathrm{~K}$. The scaling factor required to get agreement with this data is approximately 2 . (When interactions are neglected, as in Ref. 29, the scaling factor is approximately 1.5.) To reduce this discrepancy, the 
value of $t$ would need to be increased but this would also decrease the sensitivity of $I_{\sigma}(T)$ to temperature variations and, thus, $\omega_{S F}$ would need to be adjusted as well. To prove this we employ results of local density approximation calculations by Markievicz et al ${ }_{51}^{51}$ which suggest significantly bigger values for $t$ in BSCCO with a Fermi surface which is little different from the one presented in Fig. 212. Using the dispersion relation, Eq. (3), and the parameter values of Table I of Ref. 51 we find $I_{\sigma}(T=0)=267.34 \mathrm{meV}$ for the non-interacting system, well above the value of $\rho_{L}(T=0) \approx 171.53 \mathrm{meV}$ as has been extrapolated from the normal state experimental data of Ref. 27. In order to reproduce the experimentally observed temperature dependence $\rho_{L}(T) / \rho_{L}(T=0)$ we have to introduce interactions and a value $\omega_{S F}=8 \mathrm{meV}$ is found to give excellent agreement of $I_{\sigma}(T) / I_{\sigma}(T=0)$ with experiment. In this case $I_{\sigma}(T=0)=230.34 \mathrm{meV}$, still well above the experimental value and a down-scaling of $I_{\sigma}(T)$ by a factor of 0.745 is required to achieve agreement with experiment. Ultimately, a dispersion relation somewhere between Model $\mathrm{A}$ and the one reported by Markievicz et al ${ }^{51}$ and an $\omega_{S F}$ between 8 and $13 \mathrm{meV}$ will result in a $I_{\sigma}(T)$ from theory which agrees with the experimental $\rho_{L}(T)$ without scaling. However, our main aim is not to treat a specific case but to understand better the role interactions between the charge carriers can play in the OS. Interactions introduce a new energy scale into the problem, namely $\omega_{S F}$ for the NAFFL model. This energy scale is additional to the chemical potential or the hopping parameter $t$. With the values of the microscopic parameters associated with the NAFFL model just described, we proceed to compute the OS for temperatures at and below $T_{c}$. The solid squares give the continuation of the normal state curve and are presented for comparison with the solid triangles which are the equivalent results in the superconducting state. Again, superconducting state results fall below the normal state ones but they are seen to, nevertheless, increase with decreasing $T$. This occurs even if an Eliashberg formulation is used which represents a generalization of BCS theory and, in that sense, is not exotic. The mechanism is the exchange of antiferromagnetic spin fluctuations. On comparing the top frame of Fig. 目 with Fig. [5 we note that whether or not the superconducting state results keep increasing with decreasing temperature is, for a given band structure, governed by the value of $\omega_{S F}$.

In Fig. 5 we show additional results, open squares for a model normal state and open triangles for the superconducting state. Now, there is a further dramatic increase in the OS both in the normal and the superconducting state as compared to its value in the normal state at $T=T_{c}=90 \mathrm{~K}$. A detailed explanation of how these results were arrived at is required. In their analysis of optical data Carbotte et al ${ }^{39}$ found that the spin fluctuations themselves are modified when the superconducting state sets in. To carry out their analysis these authors used a simplified version of our Eliashberg Eqs. (1) which follows when the sum over $\mathbf{k}$ is changed to an energy integral as well as an angular average and the energy integral is done analytically in a constant density of states approximation for an infinite band model with interactions pinned to the Fermi surface. Here we have been more realistic but what is important for us in the work of Ref. 39 is that they find that the spin fluctuation spectrum is gaped at low energies, or at the very least loses intensity and a spin resonance or peak forms at higher energy. This readjustment in the spin susceptibility is not unexpected and is a characteristic that should be seen in any electronic mechanism for superconductivity $\stackrel{45,46.47 .48,49,50,52,53,54.55,56}{ }$ Details are not important for the present discussion beyond the fact that some adjustment of the spin susceptibility $\Im m \chi(\mathbf{q}, \omega)$ at small $\omega$ is expected, which weakens the inelastic scattering. Here we simply use the same low $\omega\left[\omega_{c}(T)\right]$ cutoff applied to Eq. (4) which was determined by E. Schachinger et al., ${ }^{49}$ through consideration of microwave data. Another approach would be to calculate the low energy gaping of the spin susceptibility from first principles but this would go beyond the scope of this work and would introduce additional uncertainties. The temperature dependence of $\omega_{c}(T)$ follows the temperature dependence of the superconducting gap with a maximum value of $24 \mathrm{meV}$. Application of this cutoff in otherwise standard Eliashberg calculations based on our Eqs. (11) yield the open triangles (superconducting state) and open squares (normal state) of Fig. [5] The physics underlying these curves has been made clear in a simple model recently studied by Knigavko et al $\stackrel{35}{n}$ These authors studied a model in which the charge carriers are coupled to a single Einstein mode of unspecified origin. What they found was that stiffening of this mode decreases the kinetic energy and hence increases the OS. This is precisely the same mechanism that is operative in Fig. [5 By applying a low frequency cutoff to the spin fluctuations in our MMP-model we are decreasing the KE. This decrease in KE, present in the underlying normal state below $T_{c}$, compensates for the increase in KE intrinsic to the superconducting transition which results from the opening of the superconducting gap. We note, however, that in our formulation the OS, at any given temperature, is always below (although not very much) its normal state value at this same temperature calculated with the spectrum with a low frequency cutoff (open triangles). But this cutoff is only operative in the superconducting state and is responsible for making the open triangles fall above the solid squares. The kinetic energy in the superconducting state with low frequency cutoff is now less than the normal state kinetic energy without cutoff. Including the feedback mechanism of the formation of the superconducting state on the spin susceptibility itself has the net effect, at zero temperature (where it is largest), of changing the sign of the KE contribution to the condensation energy from that in BCS. 


\section{CONCLUSION}

We have used the Nearly Antiferromagnetic Fermi Liquid model to study the effect of interactions on the optical sum and on the kinetic energy in tight binding bands. Comparison of normal state results with equivalent results when interactions are neglected showed that temperature variations can be strongly affected by details of the microscopic parameters involved in the spin fluctuation exchange mechanism. Behaviors are possible which can be quite different from the non interacting independent particle model. Comparison with normal state experimental data proves that the tight binding model of non interacting particles is certainly not adequate to describe properly the temperature dependence of the optical sum. (This has also been observed by Benfatto et $\left.a l{ }^{31}\right)$ Taking into account interactions between the charge carriers makes the tight binding model a viable model for the analysis of the temperature dependence of the normal state optical sum. This was demonstrated for the particular case of BSCCO and a particle interaction modeled on the NAFFL. Other models, like the one presented by Toschi et al. ${ }^{32}$ are also capable to reproduce the temperature dependence $I_{\sigma}(T) / I_{\sigma}(T=0)$ but they lack agreement with the value of the optical sum at zero temperature.

When superconductivity is considered within an Eliashberg formalism, the superconducting gap has $d$ wave symmetry as a function of momentum in the two dimensional Brillouin zone. The optical sum is found to decrease with decreasing temperature for some range of parameters characterizing the spin susceptibility but can also increase. This increase cannot necessarily be interpreted as kinetic energy driven superconductivity. In fact, in all cases considered, the optical sum is always lower, at a given temperature in the superconducting state, than it is in the corresponding normal state but, in some cases not by much. Correspondingly, the kinetic energy is increased in the superconducting state. What makes the optical sum and KE integral continue to go up (in some cases) with decreasing temperature is the fact that the interactions themselves introduce a temperature dependence in the underlying normal state.

The results just described were obtained for a fixed (i.e.: temperature independent) value of the spin susceptibility. If we consider the possibility that the spin fluctuation spectrum may itself be modified 38.39 .40 .52 .53 .54 .55 .56 by the onset of superconductivity and by temperature, even larger increases in the optical sum with decreasing temperature can be obtained. It is widely recognized that a generic feature of an electronic mechanism of superconductivity is the possible gaping of the excitation spectrum itself at small energies due to the opening of the superconducting gap. This leads to a weakening of interactions at small $\omega$ and to the so called collapse of the inelastic scattering rate 52.53 .54 .55 .56 which manifests itself as a large peak in the temperature dependence of the microwave conductivity. The weakening of the interaction in the superconducting state through the opening of a low energy gap in the spin susceptibility corresponds to a decrease in $\mathrm{KE}$ in the superconducting state which can, in the case considered, more than compensate for the intrinsic increase that accompanies the formation of Cooper pairs and, consequently, the OS rises with a larger slope in the superconducting state than in the normal state just above $T_{c}$. At $T_{c}$ there is no low energy gaping of the spin susceptibility and, therefore, the mechanism for $\mathrm{KE}$ reduction just described is not operative. In this sense our model does not describe KE driven superconductivity.

\section{Acknowledgment}

Research supported by the Natural Sciences and Engineering Research Council of Canada (NSERC) and by the Canadian Institute for Advanced Research (CIAR).
* Electronic address: schachinger@itp.tu-graz.ac.at URL: www.itp.tu-graz.ac.at/ ${ }^{\sim}$ ewald

1 T. Timusk and B. Statt, Rep. Prog. Phys. 62, 61 (1999) and references therein.

${ }^{2}$ H. Ding, T. Yokoya, J.C. Campuzano, T. Takahashi, M. Randeria, M.R. Norman, T. Mochiku, K. Kadowaki, and J. Giapintzakis, Nature (London) 382, 51 (1996).

3 A.G. Loeser, Z.-X. Shen, D.S. Dessau, D.S. Marshall, C.H. Park, P. Fournier, and A. Kapitulnik, Science 273, 325 (1996).

4 J.C. Campuzano, M.R. Norman, and M. Randeria, in The Physics of Superconductivity: Conventional and Unconventional, edited by K.H. Bennemann and J.B. Ketterson (Springer, Berlin, 2003) Vol. 2, p. 167.

${ }^{5}$ Ch. Renner, B. Revaz, J.-Y. Genoud, K. Kadowaki, and O. Fischer, Phys. Rev. Lett. 80, 149 (1998).

${ }^{6}$ V.J. Emery and S.A. Kivelson, Nature (London) 374, 134
(1995)

7 E. Carlson, V.J. Emery, S.A. Kivelson, and D. Orgard, in The Physics of Superconductivity: Conventional and Unconventional, edited by K.H. Bennemann and J.B. Ketterson (Springer, Berlin, 2003) Vol. 2, p. 275.

8 T. Eckl, W. Hanke, and E. Arrigoni, Phys. Rev. B 68, 014505 (2003).

${ }^{9}$ Q. Chen, I. Kosztin, B. Jankó, and K. Levin, Phys. Rev. Lett. 81, 4708 (1998).

10 S. Chakravarty, R.B. Laughlin, D.K. Morr, and C. Nayak, Phys. Rev. B 63, 094503 (2001).

11 B. Dòra, A. Virosztek, and K. Maki, Phys. Rev. B 65, 155119 (2002), K. Maki, B. Dòra, M. Kartsovnik, A. Virosztek, B. Korin-Hamzic, and M. Basletic, Phys. Rev. Lett. 90, 256402 (2003).

12 S. Chakravarty, H.-Y. Kee, and C. Nayak, Int. J. Mod. Phys. B 15, 2901 (2001). 
13 J.-X. Zhu, W. Kim, C.S. Ting, and J.P. Carbotte, Phys. Rev. Lett. 87, 197001 (2001).

14 X. Yang and C. Nayak, Phys. Rev. B 65, 064523 (2002).

15 P.W. Anderson, in The Theory of Superconductivity in the High- $T_{c}$ Cuprates (Princeton University Press, Princeton, 1998).

16 J.E. Hirsch, Physica C 199, 305 (1992); Physica C 201, 347 (1992).

17 J.E. Hirsch and F. Marsiglio, Phys. Rev. B 62, 15131 (2000).

18 J.E. Hirsch, Science 295, 2226 (2002).

19 Wonkee Kim and J.P. Carbotte, Phys. Rev. B 64, 104501 (2001); Phys. Rev. B 61, R11886 (2000); Phys. Rev. B 63, 140505(R) (2001).

20 S. Chakravarty, Euro. Phys. J. B 5, 337 (1998).

21 M.R. Norman and C. Pépin, Phys. Rev. B 66, 100506(R) (2002).

22 M.R. Norman and C. Pépin, Rep. Prog. Phys. 66, 1547 (2003).

23 L. Benfatto, S.G. Sharapov, and H. Beck, Eur. Phys. J. 39, 469 (2004).

24 A.E. Karakozov, E.G. Maksimov, and O.V. Dologov, Solid State Comm. 124, 119 (2002).

25 D.N. Basov, S.I. Woods, A.S. Katz, E.J. Singley, R.C. Dynes, M. Xu, D.G. Hinks, C.C. Homes, and M. Strongin, Science 283, 49 (1999).

26 A.S. Katz, S.I. Woods, E.J. Singley, T.W. Li, M. Xu, D.G. Hinks, R.C. Dynes, and D.N. Basov, Phys. Rev. B 61, 5930 (2000).

27 H.J.A. Molegraaf, C. Presura, D. van der Marel, P.H. Kes, and M. Li, Science 295, 22 (2002).

28 A.F. Santander-Syro, R.P.M.S. Lobo, W. Bontemps, Z. Konstantinovic, Z.Z. Li, and H. Raffy, Europhys. Lett. 62, 568 (2003).

29 D. van der Marel, H.J.A. Molegraaf, C. Presura, and L. Santoso, in Concepts in Electron Correlations, edited by A. Hewson and V. Zlatic (Kluwer, 2003) p. 7.

${ }^{30}$ M. Ortolani, P. Calvani, and S. Lupi, Phys. Rev. Lett. 94, 067002 (2005).

31 B.L. Benefatto, S. Sharapov, N. Andrenacci, and H. Beck, Phys. Rev. B 71, 104511 (2005).

32 A. Toschi, M. Capone, M. Ortoloani, S. Lupi, P. Calvani, and C. Castellani, cond-mat/0502528 (unpublished).

33 A.J. Millis, A. Zimmers, R.P.S.M. Lobo, and N. Bontemps, cond-mat/0411172 (unpublished).

34 A.V. Boris, N.N. Kovalera, O.V. Dolgov, T. Holden, C.T. Jin, B. Keimer, and C. Bernhard, Science 304, 708 (2004).

35 A. Knigavko, J.P. Carbotte, and F. Marsiglio, Phys. Rev. B 70, 224501 (2004).

36 A.V. Chubukov, D. Pines, and J. Schmalian, in The
Physics of Superconductivity: Conventional and Unconventional, edited by K.H. Bennemann and J.B. Ketterson (Springer-Verlag, Berlin, 2003), Vol. 2, p. 495.

37 A.J. Millis, H. Monien, and D. Pines, Phys. Rev. B 42, 167 (1990).

38 E. Schachinger, J.P. Carbotte, and D.N. Basov, Europhys. Lett. 54, 380 (2001).

39 J.P. Carbotte, E. Schachinger, and D.N. Basov, Nature (London) 401, 354 (1999).

40 E. Schachinger and J.P. Carbotte, Phys. Rev. B 62, 9054 (2000).

41 D. Branch and J.P. Carbotte, Can. J. Phys. 77, 531 (1999); J. Superconductivity 12, 667 (1999); 13, 535 (2000).

42 P. Monthoux and D. Pines, Phys. Rev. B 47, 6069 (1993); 49, 4261 (1994).

43 A. Abanov, A.V.Chubukov, and J. Schmalian, Adv. in Physics 52, 119 (2003).

44 E. Schachinger, J.J. Tu, and J.P. Carbotte. Phys. Rev. B 67, 214508 (2003).

45 E. Schachinger and J.P. Carbotte, Phys. Rev. B 64, 094501 (2001); Phys. Rev. B 65, 064514 (2002).

46 A. Abanov, A.V. Chubukov, and J. Schmalian, Phys. Rev. B 63, 180510 (2001); J. Electron Spectrosc. 117, 129 (2001).

47 J.P. Carbotte and E. Schachinger, Phys. Rev. B 69, 224501 (2004).

48 E. Schachinger and J.P. Carbotte, in: Models and Methods of High-TC Superconductivity: some Frontal Aspects, edited by J.K. Srivastava and S.M. Rao, (Nova Science, Hauppauge, NY, 2003), Vol. II, p. 73.

49 E. Schachinger, J.P. Carbotte, and F. Marsiglio, Phys. Rev. B 56, 2738 (1997).

50 P. Monthoux, A.V. Balatsky, and D. Pines, Phys. Rev. Lett. 67, 3448 (1991); Phys. Rev. B 46, 14803 (1992).

51 R.S. Markiewicz, S. Sahrakorpi, M. Lindroos, Hsin Lin, and A. Bansil, cond-mat/0503064 (unpublished) and references therein.

52 M.C. Nuss, P.M. Mankiewich, N.L. O'Malley, E.H. Westwick, and P.B. Littlewood, Phys. Rev. Lett. 66, 3305 (1991).

53 D.B. Romero, C.D. Porter, D.B. Tanner, L. Forro, D. Mandrus, L. Mihaly, C.C. Carr, and G.P. Williams, Phys. Rev. Lett. 68, 1590 (1992).

54 D.A. Bonn, P. Dosanjh, R. Liang, and W.N. Hardy, Phys. Rev. Lett. 68, 2390 (1992).

55 A. Hosseini, R. Harris, S. Kamal, P. Dosanjh, J. Preston, R. Liang, W.N. Hardy, and D.A. Bonn, Phys. Rev. B 60, 1349 (1999).

56 E.J. Nicol and J.P. Carbotte, Phys. Rev. B 44, R7741 (1991). 